\title{
ON A CLASS OF STRATEGY PAIRS IN MARKOV GAMES
}

\author{
Heinz-Uwe Kūenle, Technische Universität Cottbus
}

This contribution deals with Nash equilibria in two-person Markov games. A special class of strategy pairs is considered, so-called intimidation strategy pairs. The idea of such strategy pairs is the following one: Both players have an agreement to play some deterministic strategy pair, but if one of the players violates this agreement then the other player chooses such a strategy which is most unfavourable for the disloyal player.

We give simple conditions for the existence of intimidation strategy pairs which are also equilibrium strategy pairs. Furthermore, it can be shown that every loss of deterministic equilibrium pairs is also the equilibrium loss of an intimidation strategy pair. Recursive relations for the set of all deterministic equilibrium losses are presented. For Markov games with complete information it holds also that all effective equilibrium losses are losses of intimidation strategy pairs.

As an example the following situation is treated: 'Two persons harvest periodically from the same bounded source but every of this persons has the possibility to ruin the existence of the other person. The problem is here to find a compromise between the both persons how many of the source is allowed to take away.

The results can be generalised to $n$-person Markov games. 